\title{
Article \\ COVID-19 Patient Detection Based on Fusion of Transfer Learning and Fuzzy Ensemble Models Using CXR Images
}

\author{
Chandrakanta Mahanty ${ }^{1}$, Raghvendra Kumar ${ }^{1}$, Panagiotis G. Asteris ${ }^{2}\left(\mathbb{D}\right.$ and Amir H. Gandomi ${ }^{3, *(1)}$ \\ 1 Department of Computer Science and Engineering, GIET University, Rayagada 765022, Odisha, India; \\ chandra.mahanty@giet.edu (C.M.); raghvendra@giet.edu (R.K.) \\ 2 Computational Mechanics Laboratory, Department of Civil Engineering, School of Pedagogical and \\ Technological Education, 15122 Athens, Greece; asteris@aspete.gr \\ 3 Faculty of Engineering and Information Technology, University of Technology Sydney, Ultimo, \\ NSW 2007, Australia \\ * Correspondence: gandomi@uts.edu.au
}

Citation: Mahanty, C.; Kumar, R.; Asteris, P.G.; Gandomi, A.H. COVID-19 Patient Detection Based on Fusion of Transfer Learning and Fuzzy Ensemble Models Using CXR Images. Appl. Sci. 2021, 11, 11423. https://doi.org/10.3390/app112311423

Academic Editors: Federico Divina and Jing Jin

Received: 27 October 2021

Accepted: 29 November 2021

Published: 2 December 2021

Publisher's Note: MDPI stays neutral with regard to jurisdictional claims in published maps and institutional affiliations.

Copyright: (c) 2021 by the authors. Licensee MDPI, Basel, Switzerland. This article is an open access article distributed under the terms and conditions of the Creative Commons Attribution (CC BY) license (https:// creativecommons.org/licenses/by/ $4.0 /)$.

\begin{abstract}
The COVID-19 pandemic has claimed the lives of millions of people and put a significant strain on healthcare facilities. To combat this disease, it is necessary to monitor affected patients in a timely and cost-effective manner. In this work, CXR images were used to identify COVID-19 patients. We compiled a CXR dataset with equal number of 2313 COVID positive, pneumonia and normal CXR images and utilized various transfer learning models as base classifiers, including VGG16, GoogleNet, and Xception. The proposed methodology combines fuzzy ensemble techniques, such as Majority Voting, Sugeno Integral, and Choquet Fuzzy, and adaptively combines the decision scores of the transfer learning models to identify coronavirus infection from CXR images. The proposed fuzzy ensemble methods outperformed each individual transfer learning technique and several state-of-the-art ensemble techniques in terms of accuracy and prediction. Specifically, VGG16 + Choquet Fuzzy, GoogleNet + Choquet Fuzzy, and Xception + Choquet Fuzzy achieved accuracies of $97.04 \%, 98.48 \%$, and $99.57 \%$, respectively. The results of this work are intended to help medical practitioners achieve an earlier detection of coronavirus compared to other detection strategies, which can further save millions of lives and advantageously influence society.
\end{abstract}

Keywords: chest X-rays; COVID-19; pneumonia; transfer learning; fuzzy ensemble; GoogleNet; Xception; Majority Voting; Sugeno Integral; Choquet Fuzzy

\section{Introduction}

Coronavirus is an extremely contagious virus that mostly affects the lungs and is characterized by symptoms of cough, fever, and exhaustion. The disease has the potential to spread to the lower respiratory system, where it can potentially cause inflammation and swelling, resulting in pneumonia [1].

COVID-19 spreads by respiratory droplet production following coughing and patient contact. As a result, preventative methods, such as wearing masks, social distancing, and isolating infected people, have been implemented to deal with viral transmission thus far [2]. COVID-19 has devastated most healthcare systems around the world, causing economic damage and claiming the lives of numerous individuals. Although vaccines are being produced and administered around the globe, it will be a very long time before each human can be vaccinated. As new strains of infection arise, such worldwide vaccination will be further delayed and will lead to subsequent lockdowns. As a result, there is an even greater need for the accurate and early identification of COVID-19 to prevent viral spread.

Currently, RT-PCR remains the most utilized detection technique by health professionals, which requires samples to be collected from the upper and lower parts of the sputum and nasal cavity from people suspected of being infected with COVID-19. The RT-PCR test faces several limitations, such as the manual collection of samples from infected patients, 
reliance on patient consent, and the abilities of doctors, which could also impact the proper identification of coronavirus. Furthermore, the RT-PCR procedure is time-consuming and high cost. Because of these problems, multiple tests are necessary for proper assessment [3]. Chest radiography (CXR) is amongst the most widely-used techniques for diagnosing pneumonia globally [4]. CXR diagnosis offers the advantages of a simple detection procedure and low cost with low ionizing radiation intensity when compared to CT. CXR also exposes the patient to less radiation $[5,6]$. However, proper diagnosis using CXR images requires professional expertise and skill, thus demanding high-accuracy diagnoses via transfer learning models. Medical imaging professionals are encouraged to use these models to achieve fast and accurate results [7]. Due to the COVID-19 pandemic and the proceeding high demand for health services, the health systems risk collapse owing to the lack of health professionals and hospital beds. Other concerns are that coronavirus is highly infectious, and nurses, caretakers and physicians are the most vulnerable. Therefore, early identification of pneumonia is critical in terms of limiting the dissemination of the pandemic by isolating affected individuals [8].

CT and CXR images have been utilized as the imaging techniques for COVID-19 diagnosis in the majority of studies. In this regard, the presented study includes numerous pieces of research on various transfer learning techniques used to detect coronavirus via CXR imaging.

Nayak et al. [9] suggested a deep learning-based automated approach, named the ResNet-34 model, for the early detection of COVID-19 infection using CXR images. They compared the performance of eight pre-trained CNN models for discriminating COVID-19 instances from normal CXR images and achieved an accuracy of $98.33 \%$ for the proposed model. Dilshad et al. [10] introduced and applied a CNN-based MobileNet model on the Indian CXR dataset, achieving an accuracy of $96.33 \%$. Similarly, a CNN-based binary classification model (Haque and Abdelgawad et al. [11]), modified VGG-19 model (Bhatt et al. [12]), and a deep CNN architecture named CVDNet (Ouchicha et al. [13]) have been proposed to detect coronavirus in patients using CXR images, resulting in accuracies of $98.3 \%, 97.37 \%$, and $97.20 \%$, respectively. Other recommended models include two novel models, namely a dilated and depth-wise separable CNN by Li et al. [14], a modified AlexNet model by Kaur et al. [15], a deep CNN architecture named CoroNet based on Xception architecture by Khan et al. [16], an SVM-based hybrid differential evolution PSO model by Dixit et al. [17], and a novel MAnet based two-stage classification technique for coronavirus detection utilizing CXR images by Xu et al. [18]. Moreover, a computer-aided diagnostic combined approach based on graph $\mathrm{CNN}$ and pre-trained CNN model (Kumar et al. [19]), a deep Covix-net model (Vinod et al. [20]), new deep hybrid and deep boosted hybrid learning models (Khan et al. [21]), and a gradient weighted class activation mapping technique (Panwar et al. [22]) for coronavirus detection exhibited accuracies of $97.60 \%$, $97 \%, 98.53 \%$, and $95 \%$, respectively. Likewise, a DenseNet-201 architecture reported by Alhudhaif et al. [23], a PSO-based eXtreme Gradient Boosting model recommended by Dias Júnior et al. [24], an automatic AI-based system using majority voting ensemble techniques suggested by Chandra et al. [25], and a bi-level prediction model by Das et al. [26] exhibited respective accuracies of $94.96 \%, 98.71 \%, 91.329 \%$, and $96.74 \%$ to diagnose COVID-19. Other novel techniques to detect coronavirus include a deep LSTM model (Demir et al. [27]), Inception-v3 model based on deep CNN associated with Multi-Layered Perceptron model called CovScanNet (Sait et al. [28]), and a hybrid deep CNN technique with discrete wavelet transform features (Mostafiz et al. [29]. For COVID-19 detection in patients, Mahmud et al. [30] recommended a CovXNet model, and Ergen and Zafer et al. [31] suggested a MobileNetV2 deep CNN model with fuzzy color technique, which achieved an accuracy of $97.4 \%$ and $98.25 \%$, respectively. Recently, Dey et al. [32] proposed Choquet fuzzy classifier ensemble technique and Kundu et al. [33] proposed CNN models based on fuzzy rank and Gompertz function to detect coronavirus from CXR and CT images, respectively. Rahaman et al. [34] utilized 15 distinct deep CNN models and Manokaran et al. [35] suggested a DenseNet201 model based on three-class dataset for identification of COVID-19 patients 
with an accuracy of $89.3 \%$ and $94 \%$, respectively. When reviewing the related work, it was discovered that few researchers concentrated on fuzzy ensemble approaches paired with transfer learning models. An ensemble approach is a machine learning technique that integrates many base models to generate a single optimal prediction model. The outcomes of numerous models are integrated in order to enhance overall performance. Specifically, ensemble methods integrate numerous machine learning approaches into a single predictive model to enhance overall performance and predictions while reducing bias and variance. Furthermore, combining deep transfer learning models with fuzzy ensemble approaches will assist in increasing the accuracy and robustness of a detection system. The presented study considered the following.

1. CXR images were used to diagnose coronavirus, and a transfer learning and fuzzy ensemble fusion model was proposed for COVID-19 detection.

2. At first, we trained the VGG16, GoogleNet, and Xception base classifiers using deep transfer learning techniques. Using fully connected layers and the softmax layer, we extracted features and classified CXR images using pre-trained transfer learning models.

3. Then, using Majority Voting, Sugeno Integral, and Choquet fuzzy integral, the data prediction scores from multiple models were integrated to create the final forecasted labels that are more accurate than the separate model predictions.

4. For the application of Choquet fuzzy integral with other state-of-the-art models, such as Majority Voting and Sugeno Integral, we considered the validation accuracy of each classifier to compute the fuzzy membership values of each classifier. The advantage of this type of fusion is that it creates the final prediction of each sample using adaptive weights depending on each sample's confidence scores.

5. The performance of each pre-trained model, including VGG16, GoogleNet, and Xception combined individually with Majority Voting, Sugeno Integral, and Choquet fuzzy ensemble techniques was measured in terms of precision, recall, F1-Score, sensitivityand specificity.

The goal of this article was to use CXR images to predict coronavirus, aiding healthcare providers and radiologists in the early detection and isolation of positive coronavirus patients to halt the virus' rapid spread in vulnerable countries with limited hospital capacity and a low patient-to-doctor ratio and to prevent increasing fatality rates.

The remainder of the article is organized as follows. The preliminary models are provided in Section 2. Section 3 describes the proposed algorithm. Section 4 provides a brief explanation of the proposed model and comparison analysis. Section 5 presents the conclusion and future works.

\section{Preliminaries}

VGG16: VGG16 was proposed by Karen Simonyan et al. [36] in 2014, which was termed based on its 16 layers. VGG-16 contains different layers, such as three dense layers, two FL, five max-pooling layers, and 13 convolutional layers.

GoogleNet: With a reported performance of $93.3 \%$, GoogleNet [37] succeeds at the leading edge of the ImageNet detection and classification problem. The greater utilization of computer resources in the model is its advantage, in which a construction component is treated as an "inception module" that reflects the model's increased width and depth. This was one of the earliest $\mathrm{CNN}$ designs that diverge from the traditional method of just adding convolution and pooling layers in a sequential manner. Figure 1 illustrates the GoogleNet architecture, which is composed of nine initialization modules. GoogleNet is a 22 deep CNN that is a version of Google researchers' Inception Network that is built on the Inception architecture. In this case, we utilized the third version of the GoogleNet model, Inception V3. This version is made up of a hierarchy of sophisticated inception modules that integrate channel spatial convolution, pooling, and reprojection operations at different scales in each module. The model shrinks the parameter space by breaking spatial convolutions with bigger filter sizes $\mathrm{n} \times \mathrm{n}$ into a series of two convolutional operations with $\mathrm{n} \times 1$ and $1 \times \mathrm{n}$ filter sizes. The resultant network model is more complicated and 
deeper than AlexNet, although it has fewer features and lower computing complexity than AlexNet. GoogleNet does not employ fully-connected layers. Instead, the final convolutional map is subjected to channel-wise average activation values, and global average pooling values of the 2048 channels are utilized as the input image's feature vector.

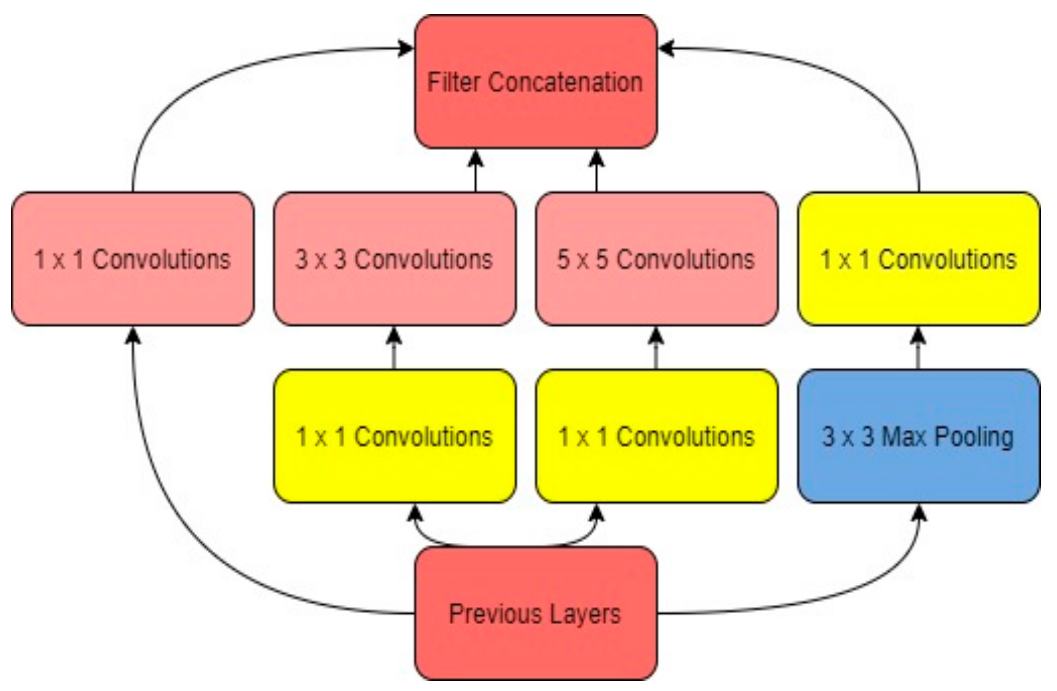

Figure 1. GoogleNet architecture.

Xception: The Xception [38] architecture includes a depth-wise separable convolution layer, convolutional layer, residual connections, and inception module. The design of Xception, which stands for "extreme inception", has 36 convolution layers that form the network's extractor base. Xception is an extension of Inception architecture by using deeply separable convolutions for standard Inception modules and, thus, has improved speed and accuracy compared to Inception, ImageNet, Inception V3, ResNet-101, ResNet-50, and VGGNet models. The Xception model contains three major sections: entry, middle, and exit flow. The data first enter the input flow, and then go through the eight-fold middle flow, and finally the exit flow. Batch normalization is accompanied by all the Convolution layers. Xception replaced the conventional starting block by expanding it and changing the various $(1 \times 1,5 \times 5,3 \times 3)$ spatial dimensions with a $(3 \times 3)$ single dimension preceded by a $(1 \times 1)$ transition to maintain computational burden. Equation(1) and Equation(2) represent the mathematical modelling of Xception:

$$
\begin{gathered}
f_{l+1}^{k}(p, q)=\sum_{x, y} f_{l}^{k}(x, y) \cdot \mathrm{e}_{1}^{\mathrm{k}}(\mathrm{u}, \mathrm{v}) \\
F_{l+2}^{k}=g_{c}\left(F_{l+2}^{k}, k_{l+1}\right)
\end{gathered}
$$

Choquet fuzzy: In this study, aggregation refers to the process of merging each classifier's performance scores into a single global score. For the said purpose, an aggregation operator was used. Normally, we consider weighted average as the aggregation operator; however, in this situation, we employed the Choquet fuzzy integral [39]. This approach has already been performed in a variety of pattern identification applications due to its benefit of capturing the unpredictability that exists in the decision values obtained as extra data via classifier fusion, which is lacking in traditional ensemble approaches. As a result, fuzzy measures are the extension of aggregation operations on a set of confidence values. Weighted values are assigned to distinct classifier combinations in these fuzzy measures. To use the Choquet fuzzy approach, we must first establish the fuzzy measure values that define the strength of each classifier. We include three different types of interaction in this section: $f\{A U B\}=f\{A\}+f\{B\}=$ sum of individual strength of $A$ and $B$ is equal to $\{A U B\}, f\{A U B\} \leq f\{A\}+f\{B\}$ and $f\{A U B\} \geq f\{A\}+f\{B\}$; Where $A$ and $B$ 
are two subsets of classifiers and mutually exclusive. $f(S)$ indicates the strength of a set of classifiers.

After obtaining a complete set of fuzzy measure scores and relevant scores for each classifier, we can utilize the Choquet fuzzy method to compute the classifiers' aggregated global score. Let $g()$ generate the fuzzy measure score of a collection of classifiers $D=\left(d_{1}, d_{2}, d_{3} \ldots . ., d_{n}\right)$, and $P=\left(p_{1}, p_{2}, p_{3} \ldots . ., p_{n}\right)$ are the individual classifier performance scores in $\mathrm{D}$. Assume $L_{i}$ is a subset of $\mathrm{D}$, such that $L_{i}=\left(d_{1}, d_{2}, d_{3} \ldots ., d_{n}\right)$ of $\mathrm{D}$, where $i$ is in between 1 and $n$. This indicates that, if $i=1$ then $L_{1}=\left(d_{1}\right)$, and if $i=3$, then $L_{3}=\left(d_{1}, d_{2}, d_{3}\right)$. Wealsoassumethat $\left(p_{1} \geq p_{2} \geq p_{3} \ldots . . \geq p_{n}\right)$, then the aggregated score may be computed using the Choquet fuzzy integral, as shown in Equation (3):

$$
\text { Choquet }_{g}\left(p_{1}, p_{2}, p_{3} \ldots . ., p_{n}\right)=p_{n}^{*} g\left(L_{n}\right)+\left(p_{n-1}-p_{n}\right)^{*} g\left(L_{n-1}\right)+\ldots+\left(p_{1}-p_{2}\right)^{*} g\left(L_{1}\right)
$$

We compute the value of $\lambda$ for determination of the fuzzy membership values using Equation (4):

$$
1+\lambda=\prod_{i=1}^{n}\left(g\left(\left\{d_{i}\right\}\right) \lambda+1\right)
$$

By obtaining the roots of the characteristic equation, we can find the value of $\lambda$. By repeating Equation (5), the fuzzy membership value of each classifier combination can be determined:

$$
g\left(\left\{d_{l}, d_{0}\right\}\right)=g\left(\left\{d_{l}\right\}\right)+g\left(\left\{d_{o}\right\}\right)+\lambda g\left(\left\{d_{l}\right\}\right) g\left(\left\{d_{o}\right\}\right)
$$

where $1 \leq 1, \mathrm{o} \leq \mathrm{n}$.

We have $p$ classifiers that are used to categorize $q$ classes when dealing with classifier combinations. Let $x_{i j}$ denote the confidence score of the $j$ th class of the $i$ th classifier. Choquet integral is now utilized for each $j$ to produce a fuzzy confidence score. In this scenario, $g\left(\left\{b_{i}\right\}\right)$ represents each classifier's fuzzy membership scores, which are empirically determined for $j=1,2,3 \ldots q, b_{i}=x_{i j}$, where $i=1,2,3 \ldots p$. In this study, however, we set the fuzzy measure $g\left(\left\{b_{i}\right\}\right)=w_{i} / w_{1}+w_{2}+w_{3} \ldots+w_{p}$, where $w_{i}$ indicates the validation accuracy of the $i$ th classifier. Even after the values of fuzzy measures have been set, the Choquet fuzzy integral can modify the weightage of each classifier by considering the decision scores supplied by the rest of the classifiers. This makes the system dynamic, as opposed to other assembly techniques that employ the majority voting and sugeno integral system.

We must first obtain fuzzy measure values for each classifier before we can use the Choquet fuzzy ensemble. The fuzzy measure values indicate the strength of each classifier and all possible classifiers. Algorithm 1 depicts the key phases of the proposed methodology.

Majority Voting: MV is a basic but effective ensemble learning approach that has been effectively utilized in a variety of industries. The fundamental concept behind MV is to use the majority voting rule to aggregate the output results from many data sources [40]. The majority voting rule is divided into three types: (i) unanimous voting, in which all classifiers must agree on a prediction; (ii) simple majority voting, in which the prediction must be supported by at least one more than half of the classifiers; and (iii) plurality or majority voting, in which the highest number of votes is considered for the ensemble decision whether the sum of those exceeds $50 \%$. In the case of independent classifier outputs, the majority voting rule combiner always improves prediction performance. The MV technique works by accumulating the labels associated with each classifier in the input image. The final label is then assigned to the class with the most labels (votes) among the classifiers. If two classes receive equal votes, we choose to assign the final label to the class with the lowest index.

Sugeno Integral: Takagi-Sugeno [41] is a fuzzy inference technique that creates a systematic strategy for generating fuzzy rules from a given input-output dataset. The inputs are blurry, but the outcome is clear. Takagi-Sugeno computes the crisp output using a weighted average. This method is computationally more efficient and may be used in conjunction with optimization and adaptive approaches. 


\section{Methodology}

Herein, we present a model that employs the deep transfer learning approach to classify CXR images as COVID-positive, negative or pneumonia. This approach first employs pre-trained transfer learning models from VGG16, GoogleNet, and Xception as feature extractors, then uses classifiers to classify the features. The classifier outputs are subsequently merged using Majority Voting, Sugeno Integral, and Choquet fuzzy integral, whereby each classifier's fuzzy measures are paired with an empirically established ratio. We used two conventional ensemble techniques (Majority Voting and Sugeno Integral) to make a comparison of the Choquet fuzzy ensemble approach for COVID-19 patient identification based on the CXR dataset. We evaluated these models for consistent performance comparison. Figure 2 depicts the proposed ensemble model.

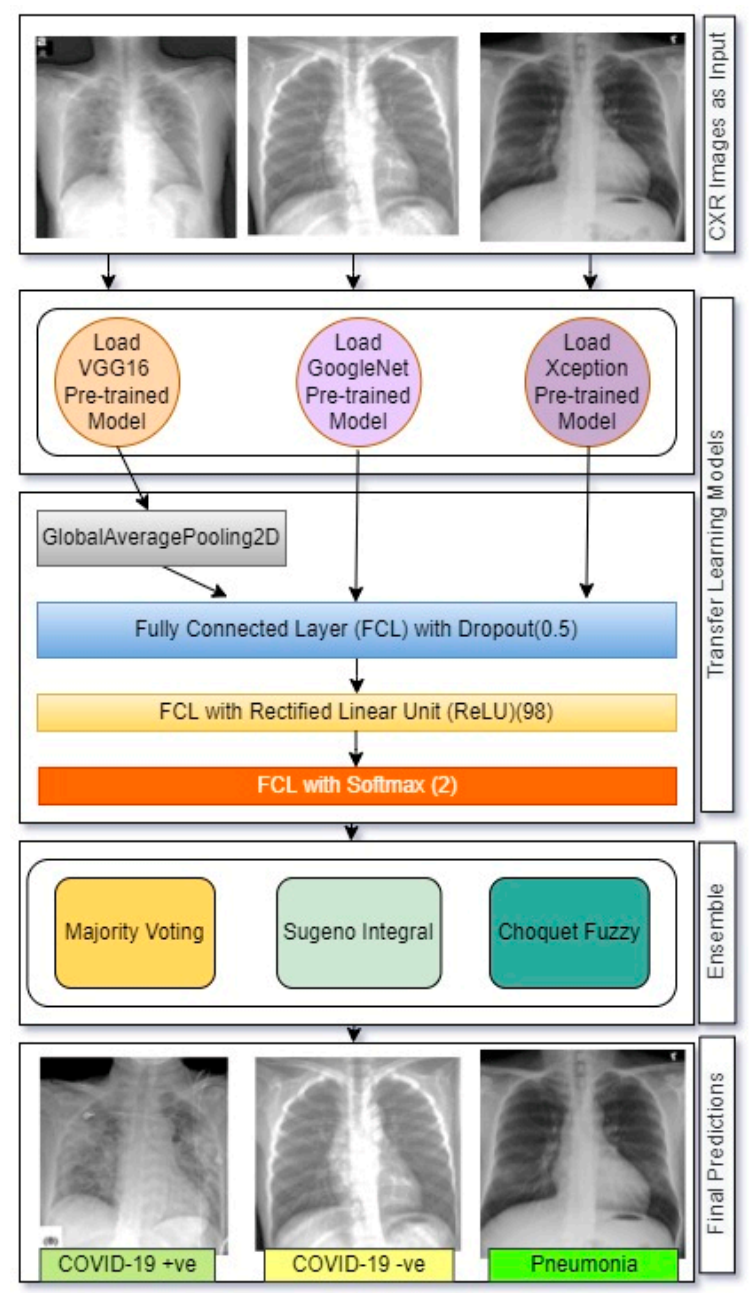

Figure 2. Working principle of VGG16, GoogleNet, and Xception pre-trained models for detection of positive, negative and pneumonia COVID-19 patients. 


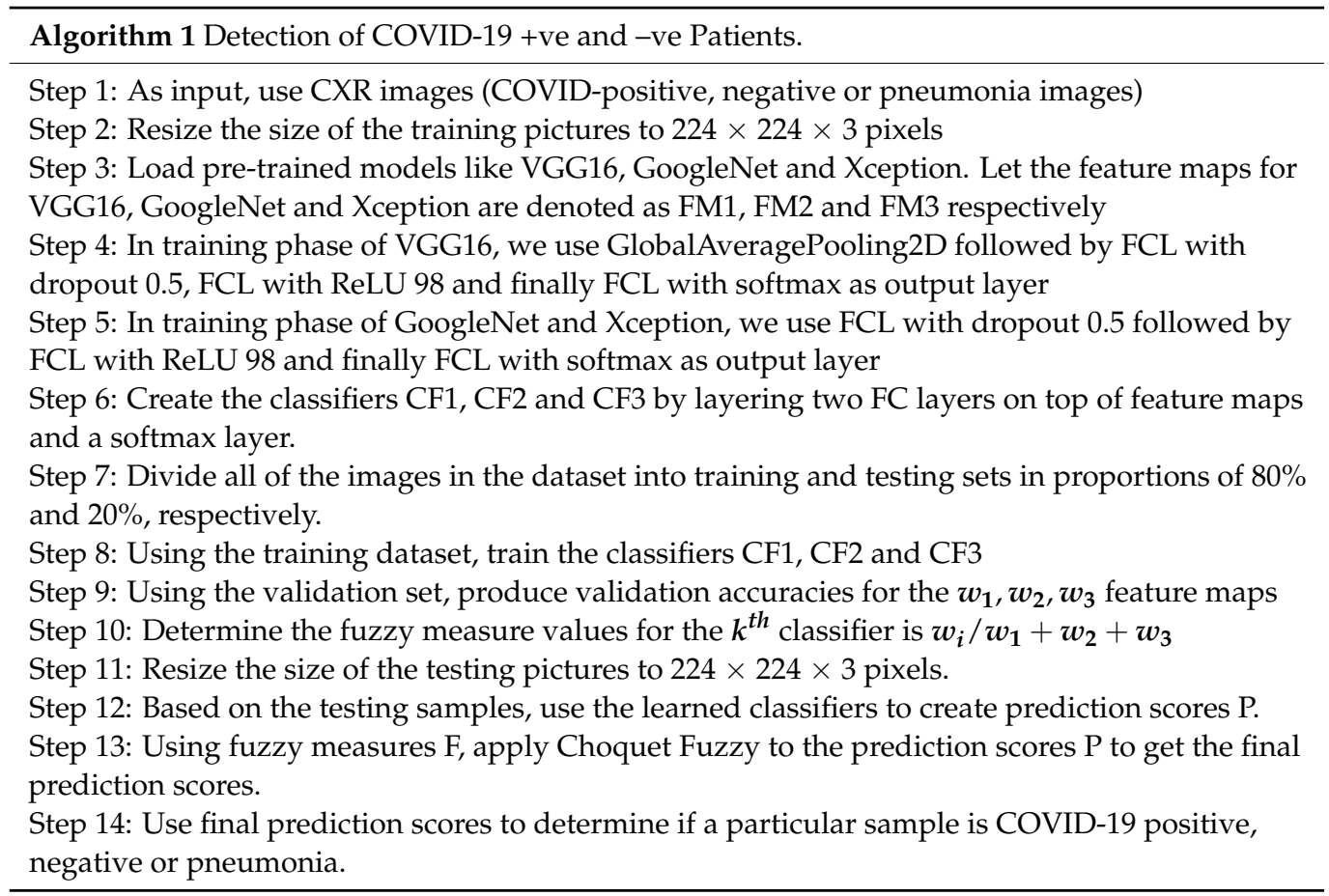

2D Global Average Pooling is employed on the spatial dimensions until each spatial dimension is equal to one. To avoid overfitting, FCL with a dropout of 0.5 is utilized. FCL combined with ReLU 98 is then utilized to enhance neural networks by accelerating training. The FCL function with the softmax function is used to normalize the outputs between 0 and 1 .

\section{Discussion}

\subsection{Experimental Setup}

The entire experiment was carried out on Google Colaboratory using a Python3 engine, a GPU as a backend, and a system RAM of 12 GB. TensorFlow 1.x was employed. For image processing, we loaded the picture as a color image with three RGB channels and transformed it into a NumPy array. On the CXR dataset, we trained all three transfer learning models with an identical set of hyperparameters. During the entry procedure, the images were scaled to $224 \times 224$ pixels with various dimensions. For training purposes, we setthe learning rate to $2 \times 10^{-4}$ with 60 epochs, which are small enough to prevent the fitting problem of the transfer learning models. An Adam optimizer was used as a compilation, and FCL with ReLU 98 was employed as the activation function for feature extraction.

\subsection{Dataset Description}

We obtained the COVID-19 CXR image dataset from Kaggle [42], which includes equal number of COVID positive, pneumonia and normal CXR images. Each class contains 2313 images. The active COVID-19, pneumonia and normal patient CXR images were used to train the Tensor Flow and Keras deep learning models to detect whether or not a person has COVID-19. The data were divided into $80 \%$ for the training set and $20 \%$ for the test sets, with the identical sets utilized for all models.

\subsection{Results and Outcomes}

Figures 3 and 4 show the accuracy and loss graphs of the transfer learning models (VGG16, GoogleNet, and Xception). Specifically, Figure 3 indicates that Xception learns faster than GoogleNet and VGG16 and excelled in the competition in terms of accuracy. Early halting and model checkpoints were used to determine the best model and stop training if there was no substantial gain in accuracy. The ImageNet weights for the 
models were set. In Figure 4, the loss for the above three models is depicted, whereby the loss rate of VGG16 was initially very high but gradually declined, while the loss rate of Xception exhibited a smooth descent. For all three models, the loss was $<0.1$. We compared the performance of the Choquet fuzzy integral-based ensemble strategy for coronavirus detection to two traditional ensemble approaches (Majority Voting and Sugeno Integral) using the current dataset.

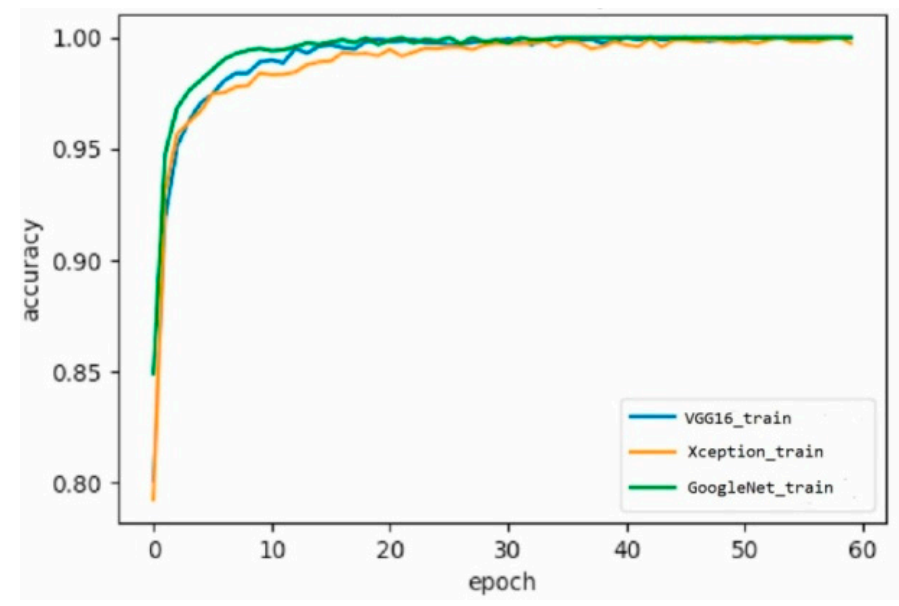

Figure 3. Accuracy graph for the training phase of VGG16, Xception and GoogleNet.

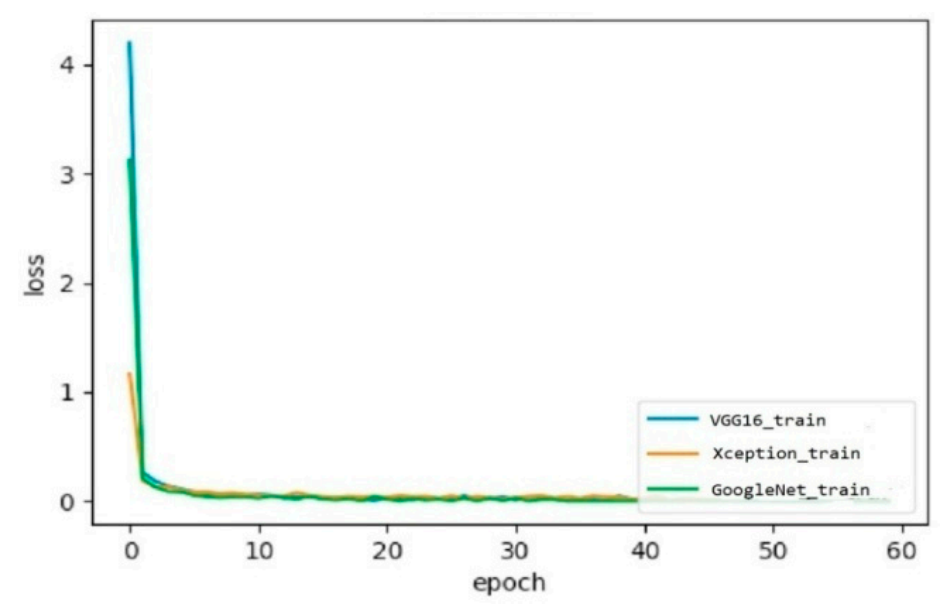

Figure 4. Loss graph for the training phase of VGG16, Xception, and GoogleNet.

\subsection{Performance Analysis}

The performance of each model was evaluated in terms of recall, precision, F1-Score, sensitivity, and specificity, and the results are represented in Table 1. Confusion matrices of the VGG16, GoogleNet, and Xception-associated fuzzy ensemble techniques are illustrated in Figures 5-7, respectively. Performance comparisons of various transfer learning models with the proposed model using CXR images are presented in Table 2. The following equations were used to calculate the performance metrics:

$$
\begin{aligned}
& \text { 1. Precision }=\frac{\text { True Positive }(T P)}{\text { True Positive }+ \text { False Positive }(F P)} \\
& \text { 2. Sensitivity or Recall }=\frac{\text { True Positive }}{\text { True Positive }+ \text { False Negative }(F N)} \\
& \text { 3. } F 1-\text { Score }=\frac{2 x(\text { Precision } x \text { Recall })}{(\text { Precision }+ \text { Recall })}
\end{aligned}
$$




$$
\begin{aligned}
& \text { 4. Specificity }=\frac{\text { True Negative }(T N)}{\text { True Negative }+ \text { False Positive }} \\
& \text { 5. Accuracy }=\frac{T P+T N}{T P+T N+F P+F N}
\end{aligned}
$$

\begin{tabular}{|c|c|c|c|c|c|c|c|c|c|c|c|}
\hline COVID +ve & CovID -ve & Pneumonia & COVID +ve & COVID -ve & Pneumonia & COVID +ve & CovID -ve & Pneumonia & COVID +ve & COVID -ve & Pneumonia \\
\hline 452 & 7 & 3 & 453 & 7 & 2 & 450 & 7 & 5 & 452 & 6 & 4 \\
\hline 8 & 442 & 12 & 8 & 442 & 12 & 8 & 443 & 11 & 10 & 442 & 10 \\
\hline 6 & 10 & 446 & 6 & 10 & 446 & 5 & 7 & 450 & 4 & 7 & 451 \\
\hline
\end{tabular}

Figure 5. Confusion matrices of VGG16 and associated fuzzy ensemble techniques. VGG16, VGG16 + Majority Voting, VGG16 + Sugeno Integral, and VGG16 + Choquet Fuzzy achieved an accuracy of 96.68\%, 96.75\%, 96.90\%, and 97.04\%, respectively.

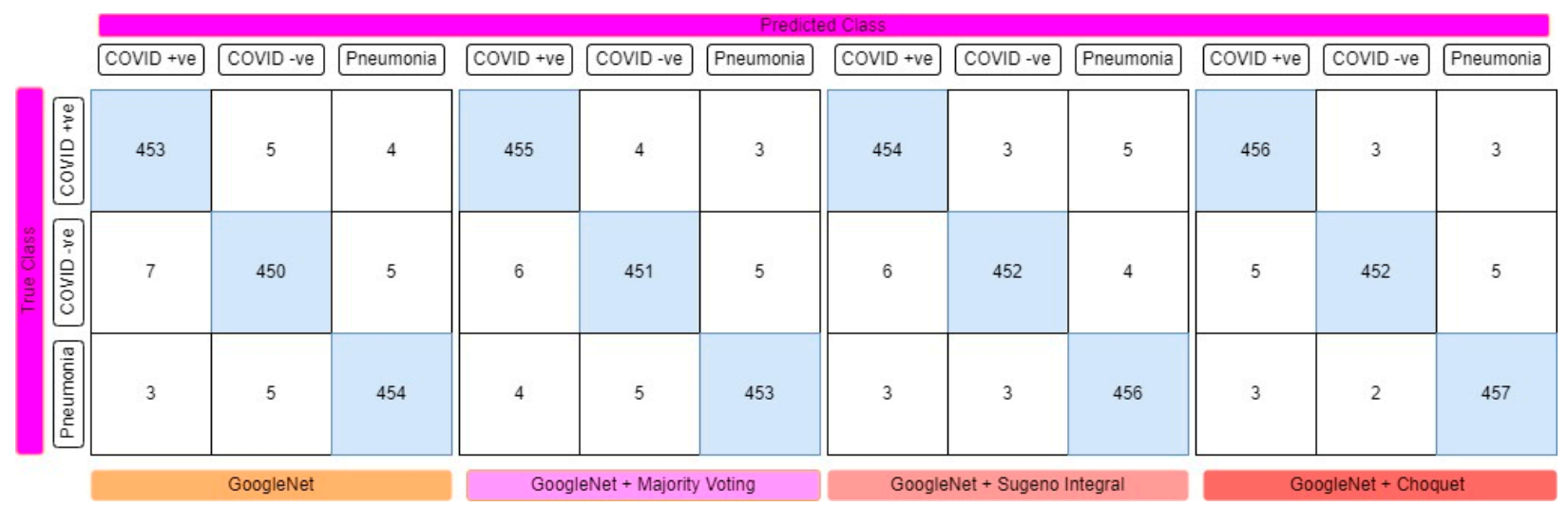

Figure 6. Confusion matrices of GoogleNet and associated fuzzy ensemble techniques. GoogleNet, GoogleNet + Majority Voting, GoogleNet + Sugeno Integral, and GoogleNet + Choquet Fuzzy achieved an accuracy of 97.91\%, 98.05\%, 98.27\%, and $98.48 \%$, respectively.

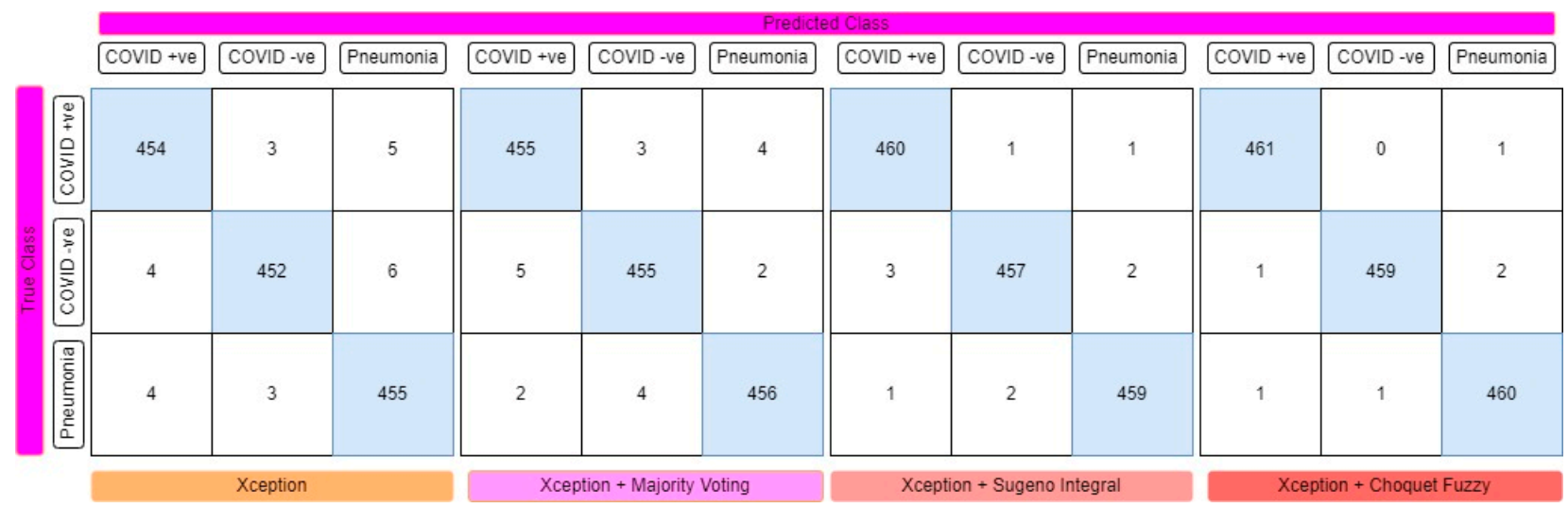

Figure 7. Confusion matrices of Xception and associated fuzzy ensemble techniques. Xception, Xception + Majority Voting, Xception + Sugeno Integral, and Xception + Choquet Fuzzy achieved an accuracy of 98.20\%, 98.56\%, 99.28\%, and $99.57 \%$, respectively. 
Table 1. Performance metrics for various transfer learning models associated with fuzzy ensemble techniques.

\begin{tabular}{|c|c|c|c|c|c|c|}
\hline Models & Class & Precision (\%) & $\begin{array}{l}\text { Sensitivity } \\
(\%)\end{array}$ & $\begin{array}{l}\text { Specificity } \\
(\%)\end{array}$ & F1-Score (\%) & Accuracy (\%) \\
\hline \multirow{3}{*}{ VGG16 } & COVID +ve & 0.970 & 0.978 & 0.985 & 0.974 & \multirow{3}{*}{96.68} \\
\hline & COVID -ve & 0.963 & 0.957 & 0.982 & 0.960 & \\
\hline & Pneumonia & 0.967 & 0.965 & 0.984 & 0.966 & \\
\hline \multirow{3}{*}{$\begin{array}{l}\text { VGG16 + } \\
\text { Majority Voting }\end{array}$} & COVID +ve & 0.970 & 0.981 & 0.985 & 0.975 & \multirow{3}{*}{96.75} \\
\hline & COVID -ve & 0.963 & 0.957 & 0.982 & 0.960 & \\
\hline & Pneumonia & 0.970 & 0.965 & 0.985 & 0.967 & \\
\hline \multirow{3}{*}{$\begin{array}{l}\text { VGG16 + } \\
\text { Sugeno Integral }\end{array}$} & COVID +ve & 0.972 & 0.974 & 0.986 & 0.973 & \multirow{3}{*}{96.90} \\
\hline & COVID -ve & 0.969 & 0.959 & 0.985 & 0.964 & \\
\hline & Pneumonia & 0.966 & 0.974 & 0.983 & 0.970 & \\
\hline \multirow{3}{*}{$\begin{array}{l}\text { VGG16 + } \\
\text { Choquet Fuzzy }\end{array}$} & COVID +ve & 0.970 & 0.978 & 0.985 & 0.974 & \multirow{3}{*}{97.04} \\
\hline & COVID -ve & 0.971 & 0.957 & 0.986 & 0.964 & \\
\hline & Pneumonia & 0.970 & 0.976 & 0.985 & 0.973 & \\
\hline \multirow{3}{*}{ GoogleNet } & COVID +ve & 0.978 & 0.981 & 0.989 & 0.979 & \multirow{3}{*}{97.91} \\
\hline & COVID -ve & 0.978 & 0.974 & 0.989 & 0.976 & \\
\hline & Pneumonia & 0.981 & 0.983 & 0.990 & 0.982 & \\
\hline \multirow{3}{*}{$\begin{array}{l}\text { GoogleNet + } \\
\text { Majority Voting }\end{array}$} & COVID +ve & 0.978 & 0.985 & 0.989 & 0.982 & \multirow{3}{*}{98.05} \\
\hline & COVID -ve & 0.980 & 0.976 & 0.990 & 0.978 & \\
\hline & Pneumonia & 0.983 & 0.981 & 0.991 & 0.982 & \\
\hline \multirow{3}{*}{$\begin{array}{l}\text { GoogleNet + } \\
\text { Sugeno Integral }\end{array}$} & COVID +ve & 0.981 & 0.983 & 0.990 & 0.982 & \multirow{3}{*}{98.27} \\
\hline & COVID -ve & 0.987 & 0.978 & 0.994 & 0.983 & \\
\hline & Pneumonia & 0.981 & 0.987 & 0.990 & 0.984 & \\
\hline \multirow{3}{*}{$\begin{array}{l}\text { GoogleNet }+ \\
\text { Choquet Fuzzy }\end{array}$} & COVID +ve & 0.983 & 0.987 & 0.991 & 0.985 & \multirow{3}{*}{98.48} \\
\hline & COVID -ve & 0.989 & 0.978 & 0.995 & 0.984 & \\
\hline & Pneumonia & 0.983 & 0.989 & 0.991 & 0.986 & \\
\hline \multirow{3}{*}{ Xception } & COVID +ve & 0.983 & 0.983 & 0.991 & 0.983 & \multirow{3}{*}{98.20} \\
\hline & COVID -ve & 0.987 & 0.978 & 0.994 & 0.983 & \\
\hline & Pneumonia & 0.976 & 0.985 & 0.988 & 0.981 & \\
\hline \multirow{3}{*}{$\begin{array}{l}\text { Xception + } \\
\text { Majority Voting }\end{array}$} & COVID +ve & 0.985 & 0.985 & 0.992 & 0.985 & \multirow{3}{*}{98.56} \\
\hline & COVID -ve & 0.985 & 0.985 & 0.992 & 0.985 & \\
\hline & Pneumonia & 0.987 & 0.987 & 0.994 & 0.987 & \\
\hline \multirow{3}{*}{$\begin{array}{l}\text { Xception + } \\
\text { Sugeno Integral }\end{array}$} & COVID +ve & 0.991 & 0.996 & 0.996 & 0.994 & \multirow{3}{*}{99.28} \\
\hline & COVID -ve & 0.993 & 0.989 & 0.997 & 0.991 & \\
\hline & Pneumonia & 0.994 & 0.994 & 0.997 & 0.994 & \\
\hline \multirow{3}{*}{ Proposed Model } & COVID +ve & 0.996 & 0.998 & 0.998 & 0.997 & \multirow{3}{*}{99.57} \\
\hline & COVID -ve & 0.998 & 0.994 & 0.999 & 0.996 & \\
\hline & Pneumonia & 0.994 & 0.996 & 0.997 & 0.995 & \\
\hline
\end{tabular}


Table 2. Performance comparisons of various transfer learning models with the suggested model using CXR images.

\begin{tabular}{lll}
\hline Authors & Best Methodology & Accuracy (\%) \\
\hline Sethy et al. [43] & ResNet50+ SVM & $95.38 \%$ \\
\hline Narin et al. [44] & Deep CNN; ResNet-50 & $98 \%$ \\
\hline Civit et al. [45] & K-Nearest Neighbour & $98.09 \%$ \\
\hline Elaziz et al. [46] & Convolutional Neural Network & $93.2 \%$ \\
\hline Mahmud et al. [30] & Neural Search Architecture & $97 \%$ \\
\hline Ucar et al. [47] & Network-Type CNN & $96.3 \%$ \\
\hline Martínez et al. [48] & VGG19 & $97 \%$ \\
\hline Vaid et al. [49] & SqueezeNet & $95 \%$ \\
\hline Khan et al. [21] & VGG16 & $98.53 \%$ \\
\hline Karakanis et al. [50] & Deep Boosted Hybrid Learning & $98.7 \%$ \\
\hline Elpeltagy et al. [51] & Binary-Classification with CGANs & $97.1 \%$ \\
\hline Júnior et al. [24] & CNN with pre-trained ResNet50 & $98.71 \%$ \\
\hline Rahaman et al. [34] & VGG19 and XGBoost + PSO & $89.3 \%$ \\
\hline Manokaran et al. [35] & 15 distinct deep CNN models & $94 \%$ \\
\hline Iraji et al. [52] & BenseNet201 model & $99.43 \%$ \\
\hline Apostolopoulos et al. [53] & MobileNetV2 & $94.72 \%$ \\
\hline Proposed Model & Xception + Choquet Fuzzy & $99.57 \%$ \\
\hline
\end{tabular}

\section{Conclusions and Future Work}

With the growing threat of new coronavirus strains throughout the world, early diagnosis of COVID-19 is critical especially due to the global scarcity of healthcare facilities. In this work, we propose a hybrid fuzzy ensemble-based method for detecting COVID-19-positive patients using CXR images. Ensemble learning can improve the overall performance by combining the prominent properties of its constituent models, resulting in better predictions than the individual contributing models. Moreover, ensemble models can enhance performance by lowering the variance of prediction errors by adding some bias to compete with base learners. In the proposed work, we used various transfer learning models, which were separately trained to make independent predictions. The models were then integrated to forecast a class value, utilizing a novel approach combining Majority Voting, Sugeno Integral, and Choquet Fuzzy. A publicly accessible database containing 6939 CXR pictures was employed to assess the effectiveness of the proposed method. The data were divided into training and test sets in proportions of $80 \%$ and $20 \%$, respectively. The proposed method, Xception + Choquet Fuzzy ensemble methods, obtained a better accuracy of $99.57 \%$ compared to other transfer learning models. The suggested methodology failed to categorize $0.43 \%$ of test data, which may be due to the relatively poor performance of the individual transfer learning models. As a result, creating or selecting better individual transfer learning models may help to amend this issue. In addition, only three basic transfer learning models were applied in the aforementioned ensemble method. Therefore, adding more classifier techniques might be an additional option to improve the current method. To demonstrate its robustness across various domains, the proposed model might be applied to various classification-based image analyses. Such advancements will aid radiologists to develop a graphical user interface (GUI) for recognizing COVID-19 to keep up with the disease's various stages of progression and imagistic patterns. 
Author Contributions: Conceptualization, R.K. and C.M.; methodology, P.G.A. and A.H.G. software, R.K.; validation, C.M. and A.H.G.; formal analysis, P.G.A.; investigation, A.H.G.; resources, C.M.; data curation, R.K.; writing—original draft preparation, A.H.G.; writing—review and editing, A.H.G.; visualization, P.G.A. All authors have read and agreed to the published version of the manuscript.

Funding: This research received no external funding.

Conflicts of Interest: The authors declare no conflict of interest.

\section{References}

1. Kim, D.; Lee, J.-Y.; Yang, J.-S.; Kim, J.W.; Kim, V.N.; Chang, H. The Architecture of SARS-CoV-2 Transcriptome. Cell 2020, 181, 914-921.e10. [CrossRef]

2. Silva, P.C.; Batista, P.V.; Lima, H.S.; Alves, M.A.; Guimarães, F.G.; Silva, R.C. COVID-ABS: An agent-based model of COVID-19 epidemic to simulate health and economic effects of social distancing interventions. Chaos Solitons Fractals 2020, $139,110088$. [CrossRef]

3. Li, Y.; Yao, L.; Li, J.; Chen, L.; Song, Y.; Cai, Z.; Yang, C. Stability issues of RT-PCR testing of SARS-CoV-2 for hospitalized patients clinically diagnosed with COVID-19. J. Med.Virol. 2020, 92, 903-908. [CrossRef] [PubMed]

4. Jaiswal, A.K.; Tiwari, P.; Kumar, S.; Gupta, D.; Khanna, A.; Rodrigues, J.J. Identifying pneumonia in chest X-rays: A deep learning approach. Measurement 2019, 145, 511-518. [CrossRef]

5. Gaál, G.; Maga, B.; Lukács, A. Attention u-net based adversarial architectures for chest x-ray lung segmentation. arXiv 2020, arXiv:2003.10304.

6. Smith-Bindman, R.; Lipson, J.; Marcus, R.; Kim, K.P.; Mahesh, M.; Gould, R.; De González, A.B.; Miglioretti, D.L. Radiation dose associated with common computed tomography examinations and the associated lifetime attributable risk of cancer. Arch. Intern. Med. 2009, 169, 2078-2086. [CrossRef]

7. Minaee, S.; Kafieh, R.; Sonka, M.; Yazdani, S.; Soufi, G.J. Deep-COVID: Predicting COVID-19 from chest X-ray images using deep transfer learning. Med. Image Anal. 2020, 65, 101794. [CrossRef]

8. Sen-Crowe, B.; Sutherland, M.; McKenney, M.; Elkbuli, A. A closer look into global hospital beds capacity and resource shortages during the COVID-19 pandemic. J. Surg. Res. 2021, 260, 56-63. [CrossRef]

9. Nayak, S.R.; Nayak, D.R.; Sinha, U.; Arora, V.; Pachori, R.B. Application of deep learning techniques for detection of COVID-19 cases using chest X-ray images: A comprehensive study. Biomed. Signal Process. Control. 2021, 64, 102365. [CrossRef]

10. Dilshad, S.; Singh, N.; Atif, M.; Hanif, A.; Yaqub, N.; Farooq, W.; Ahmad, H.; Chu, Y.-M.; Masood, M.T. Automated image classification of chest X-rays of COVID-19 using deep transfer learning. Results Phys. 2021, 28, 104529. [CrossRef]

11. Haque, K.F.; Abdelgawad, A. A deep learning approach to detect COVID-19 patients from chest X-ray images. AI 2020, 1, 418-435. [CrossRef]

12. Bhatt, A.; Ganatra, A.; Kotecha, K. COVID-19 pulmonary consolidations detection in chest X-ray using progressive resizing and transfer learning techniques. Heliyon 2021, 7, e07211. [CrossRef] [PubMed]

13. Ouchicha, C.; Ammor, O.; Meknassi, M. CVDNet: A novel deep learning architecture for detection of coronavirus (COVID-19) from chest x-ray images. Chaos Solitons Fractals 2020, 140, 110245. [CrossRef]

14. Li, X.; Zhai, M.; Sun, J. DDCNNC: Dilated and depthwise separable convolutional neural Network for diagnosis COVID-19 via chest X-ray images. Int. J. Cogn. Comput. Eng. 2021, 2, 71-82. [CrossRef]

15. Kaur, M.; Kumar, V.; Yadav, V.; Singh, D.; Kumar, N.; Das, N.N. Metaheuristic-based Deep COVID-19 screening model from chest X-ray images. J. Health Eng. 2021, 2021, 1-9. [CrossRef]

16. Khan, A.I.; Shah, J.L.; Bhat, M.M. CoroNet: A deep neural network for detection and diagnosis of COVID-19 from chest X-ray images. Comput. Methods Programs Biomed. 2020, 196, 105581. [CrossRef]

17. Dixit, A.; Mani, A.; Bansal, R. CoV2-Detect-Net: Design of COVID-19 prediction model based on hybrid DE-PSO with SVM using chest X-ray images. Inf. Sci. 2021, 571, 676-692. [CrossRef] [PubMed]

18. Xu, Y.; Lam, H.-K.; Jia, G. MANet: A two-stage deep learning method for classification of COVID-19 from Chest X-ray images. Neurocomputing 2021, 443, 96-105. [CrossRef]

19. Kumar, A.; Tripathi, A.R.; Satapathy, S.C.; Zhang, Y.D. SARS-Net: COVID-19 Detection from Chest X-Rays by Combining Graph Convolutional Network and Convolutional Neural Network. Pattern Recognit. 2020, 122, 108255. [CrossRef] [PubMed]

20. Vinod, D.N.; Jeyavadhanam, B.R.; Zungeru, A.M.; Prabaharan, S. Fully automated unified prognosis of Covid-19 chest X-ray/CT scan images using Deep Covix-Net model. Comput. Biol. Med. 2021, 136, 104729. [CrossRef]

21. Khan, S.H.; Sohail, A.; Khan, A.; Hassan, M.; Lee, Y.S.; Alam, J.; Basit, A.; Zubair, S. COVID-19 detection in chest X-ray images using deep boosted hybrid learning. Comput. Biol. Med. 2021, 137, 104816. [CrossRef]

22. Panwar, H.; Gupta, P.; Siddiqui, M.K.; Morales-Menendez, R.; Bhardwaj, P.; Singh, V. A deep learning and grad-CAM based color visualization approach for fast detection of COVID-19 cases using chest X-ray and CT-Scan images. Chaos Solitons Fractals 2020, 140, 110190. [CrossRef]

23. Alhudhaif, A.; Polat, K.; Karaman, O. Determination of COVID-19 pneumonia based on generalized convolutional neural network model from chest X-ray images. Expert Syst. Appl. 2021, 180, 115141. [CrossRef] 
24. Júnior, D.A.D.; da Cruz, L.B.; Diniz, J.O.B.; da Silva, G.L.F.; Junior, G.B.; Silva, A.C.; de Paiva, A.C.; Nunes, R.A.; Gattass, M. Automatic method for classifying COVID-19 patients based on chest X-ray images, using deep features and PSO-optimized XGBoost. Expert Syst. Appl. 2021, 183, 115452. [CrossRef]

25. Chandra, T.B.; Verma, K.; Singh, B.K.; Jain, D.; Netam, S.S. Coronavirus disease (COVID-19) detection in Chest X-Ray images using majority voting based classifier ensemble. Expert Syst. Appl. 2021, 165, 113909. [CrossRef] [PubMed]

26. Das, S.; Roy, S.D.; Malakar, S.; Velásquez, J.D.; Sarkar, R. Bi-Level prediction model for screening COVID-19 patients using chest X-ray images. Big Data Res. 2021, 25, 100233. [CrossRef]

27. Demir, F. DeepCoroNet: A deep LSTM approach for automated detection of COVID-19 cases from chest X-ray images. Appl. Soft Comput. 2021, 103, 107160. [CrossRef] [PubMed]

28. Sait, U.; Lal K.V., G.; Shivakumar, S.; Kumar, T.; Bhaumik, R.; Prajapati, S.; Bhalla, K.; Chakrapani, A. A deep-learning based multimodal system for Covid-19 diagnosis using breathing sounds and chest X-ray images. Appl. Soft Comput. 2021, $109,107522$. [CrossRef]

29. Mostafiz, R.; Uddin, M.S.; Alam, N.-A.; Reza, M.; Rahman, M.M. Covid-19 detection in chest X-ray through random forest classifier using a hybridization of deep CNN and DWT optimized features. J. King Saud Univ. Comput. Inf. Sci. 2020. [CrossRef]

30. Mahmud, T.; Rahman, A.; Fattah, S.A. CovXNet: A multi-dilation convolutional neural network for automatic COVID-19 and other pneumonia detection from chest X-ray images with transferable multi-receptive feature optimization. Comput. Biol. Med. 2020, 122, 103869. [CrossRef] [PubMed]

31. Toğaçar, M.; Ergen, B.; Cömert, Z. COVID-19 detection using deep learning models to exploit Social Mimic Optimization and structured chest X-ray images using fuzzy color and stacking approaches. Comput. Biol. Med. 2020, 121, 103805. [CrossRef]

32. Dey, S.; Bhattacharya, R.; Malakar, S.; Mirjalili, S.; Sarkar, R. Choquet Fuzzy Integral-based Classifier Ensemble Technique for COVID-19 Detection. Comput. Biol. Med. 2021, 135, 104585. [CrossRef]

33. Kundu, R.; Basak, H.; Singh, P.K.; Ahmadian, A.; Ferrara, M.; Sarkar, R. (). Fuzzy rank-based fusion of CNN models using Gompertz function for screening COVID-19 CT-scans. Sci. Rep. 2021, 11, 14133. [CrossRef] [PubMed]

34. Rahaman, M.M.; Li, C.; Yao, Y.; Kulwa, F.; Rahman, M.A.; Wang, Q.; Qi, S.; Kong, F.; Zhu, X.; Zhao, X. Identification of COVID-19 samples from chest $X$-Ray images using deep learning: A comparison of transfer learning approaches. J. X-ray Sci. Technol. 2020, 28, 821-839. [CrossRef]

35. Manokaran, J.; Zabihollahy, F.; Hamilton-Wright, A.; Ukwatta, E. Detection of COVID-19 from chest x-ray images using transfer learning. J. Med. Imaging 2021, 8, 017503. [CrossRef] [PubMed]

36. Simonyan, K.; Zisserman, A. Very Deep Convolutional Networks for Large-Scale Image Recognition. arXiv 2014, arXiv:1409.1556.

37. Szegedy, C.; Liu, W.; Jia, Y.; Sermanet, P.; Reed, S.; Anguelov, D.; Erhan, D.; Vanhoucke, V.; Rabinovich, A. Going deeper with convolutions. In Proceedings of the IEEE Conference on Computer Vision and Pattern Recognition, Boston, MA, USA, 7-12 June 2015; pp. 1-9. [CrossRef]

38. Chollet, F. Xception: Deep learning with depthwise separable convolutions. In Proceedings of the 2017 IEEE Conference on Computer Vision and Pattern Recognition (CVPR), Honolulu, HI, USA, 21-26 July 2017; pp. 1800-1807. [CrossRef]

39. Murofushi, T.; Sugeno, M. An interpretation of fuzzy measures and the Choquet integral as an integral with respect to a fuzzy measure. Fuzzy Sets Syst. 1989, 29, 201-227. [CrossRef]

40. Kittler, J.; Hatef, M.; Duin, R.P.W.; Matas, J. On combining classifiers. IEEE Trans. Pattern Anal. Mach. Intell. 1998, 20, 226-239. [CrossRef]

41. Sugeno, M. An introductory survey of fuzzy control. Inf. Sci. 1985, 36, 59-83. [CrossRef]

42. COVID19_Pneumonia_Normal_Chest_Xray_PA_Dataset. 2021. Available online: https://www.kaggle.com/amanullahasraf/ covid19-pneumonia-normal-chest-xray-pa-dataset (accessed on 24 August 2021).

43. Sethy, P.K.; Behera, S.K.; Ratha, P.K.; Biswas, P. Detection of coronavirus disease (COVID-19) based on deep features. Int. J. Math. Eng. Manag. Sci. 2020, 5, 643-651. [CrossRef]

44. Narin, A.; Kaya, C.; Pamuk, Z. Automatic detection of coronavirus disease (COVID-19) using X-ray images and deep convolutional neural networks. arXiv 2014, arXiv:2003.10849. [CrossRef]

45. Civit-Masot, J.; Luna-Perejón, F.; Morales, M.D.; Civit, A. Deep learning system for COVID-19 diagnosis aid using X-ray pulmonary images. Appl. Sci. 2020, 10, 4640. [CrossRef]

46. Elaziz, M.A.; Hosny, K.M.; Salah, A.; Darwish, M.M.; Lu, S.; Sahlol, A.T. New machine learning method for image-based diagnosis of COVID-19. PLoS ONE 2020, 15, e0235187. [CrossRef]

47. Ucar, F.; Korkmaz, D. COVIDiagnosis-Net: Deep Bayes-SqueezeNet based diagnosis of the coronavirus disease 2019 (COVID-19) from X-ray images. Med. Hypotheses 2020, 140, 109761. [CrossRef] [PubMed]

48. Martinez, F.; Martínez, F.; Jacinto, E. Performance evaluation of the NASNet convolutional network in the automatic identification of COVID-19. Int. J. Adv. Sci. Eng. Inf. Technol. 2020, 10, 662-667. [CrossRef]

49. Vaid, S.; Kalantar, R.; Bhandari, M. Deep learning COVID-19 detection bias: Accuracy through artificial intelligence. Int. Orthop. 2020, 44, 1539-1542. [CrossRef] [PubMed] 
50. Karakanis, S.; Leontidis, G. Lightweight deep learning models for detecting COVID-19 from chest X-ray images. Comput. Biol. Med. 2021, 130, 104181. [CrossRef] [PubMed]

51. Elpeltagy, M.; Sallam, H. Automatic prediction of COVID- 19 from chest images using modified ResNet50. Multimed. Tools Appl. 2021, 4, 1-13. [CrossRef]

52. Iraji, M.S.; Feizi-Derakhshi, M.-R.; Tanha, J. COVID-19 detection using deep convolutional neural networks and binary differential algorithm-based feature selection from X-ray images. Complexity 2021, 2021, 1-10. [CrossRef]

53. Apostolopoulos, I.D.; Mpesiana, T.A. Covid-19: Automatic detection from X-ray images utilizing transfer learning with convolutional neural networks. Phys. Eng. Sci. Med. 2020, 43, 635-640. [CrossRef] 